\title{
THE ANTIBODIES INVOLVED IN THE HUMAN IMMUNE RESPONSE TO LEPTOSPIRAL INFECTION
}

\author{
B. AdLER AND S. FAINE \\ Department of Microbiology, Monash University Medical School, Alfred Hospital, \\ Prahran, 3181 Victoria, Australia
}

THE agglutinating antibody response in human leptospirosis has been extensively studied and used for diagnosis (Schüffner and Mochtar, 1926-27). The microscopic agglutination (MA) test remains the definitive diagnostic test (Turner, 1968).

Apart from the agglutinin response, several workers have studied antibody reactions with erythrocyte-sensitising substances (ESS) extracted from leptospires. Chang and McComb (1954) reported complete cross-reactivity among rabbit antisera against ESS from five strains, while Cox (1955) found very broad reactivity between ESS and human sera in a haemolytic (HL) test. The first detailed study of the human antibody response to this ESS (HL) antigen was reported by Cox, Alexander and Murphy (1957) who examined the sera of 190 leptospirosis patients from Malaya, from 79 of whom leptospires were isolated; the results revealed infections due to at least 24 different serovars. All but three of the patients produced antibodies against $\mathrm{HL}$ antigen extracted from the CDC strain of the non-pathogenic Biflexa complex, thus suggesting that the $\mathrm{HL}$ antigen reacted with a genus-wide specificity.

There has been little information published on the class of immunoglobulin involved in the human antibody response to leptospiral infection.

Hartmann et al., (1964) investigated, by analytical ultracentrifugation, the antibodies produced by a single patient infected with serovar australis, and found them to be macroglobulins. Pike et al. (1965) found that in four patients in whom serological tests had indicated infection with serovars canicola, grippotyphosa or pomona, the predominant homologous agglutinins were IgM. Sera at different stages of illness were not examined, but in one patient with serovar pomona infection, IgM agglutinins still predominated in serum taken 2 months after the onset of illness. In contrast, the agglutinins that cross-reacted with serovar sejroe were mainly IgG. Conclusions cannot be drawn about which of the agglutinins in the study were homologous because all four patients possessed agglutinins to two or more serovars, differing in their titres by only one dilution.

Tong et al. (1971) found that no antibody could be shown by the MA, HL or complementfixation tests in the IgG fractions of serum samples taken up to 3 weeks after the onset of illness in three cases-two proved by isolation of leptospires-of human infection with serovar autumnalis, pyrogenes or bataviae; however, samples from the later stages of infection were not examined. Palit and Gulasekharam (1973) reported that, in two patients with leptospirosis, antibodies reacting with $\mathrm{HL}$ antigen extracted from serovar patoc (Biflexa complex)

Received 21 Nov. 1977; accepted 7 Feb. 1978.

A part of this paper was presented in 1975 to the National Symposium on Leptospirosis, Leptospira and other Spirochaeta, in Bucharest, Romania.

J. MED. MICROBIOL.-VOL. 11 (1978) 
were restricted to the IgM fractions; neither the intervals between the onset of illness and the collection of serum samples nor the agglutinin titres of the samples were specified.

Sulzer et al. (1975) found that 42 out of 229 sera containing leptospiral antibodies against 11 different serovars retained antibody activity after reduction with 2-mercaptoethanol (2-ME). Sera with titres of $\geqslant 3200$ after reduction were all convalescent-stage sera. In contrast, all sera lost their activity against $\mathrm{HL}$ antigen after reduction; this suggests that IgM plays a dominant role in the HL reaction.

There have been no reports on the protective capacity of the different antibody classes produced in human leptospiral infection, nor investigations to determine the antigens or structural components of the leptospires against which antibodies are directed. Past studies have either not made use of the sera of patients from whom the infecting serovar was isolated and from whom serial specimens were taken (Pike et al., 1965), or have relied solely on 2-ME sensitivity as a criterion of IgM activity (Sulzer et al., 1975).

In this study serial specimens of serum were available from patients from whom the infecting serovar had been cultivated and identified. The objectives were $(a)$ to investigate the $\operatorname{IgM}$ and $\operatorname{IgG}$ response with respect to agglutinins and to antibodies against erythrocyte-sensitising F4 antigen (Faine, Adler and Palit, 1974), and (b) to determine the relationship between agglutinin titre and the capacity of human serum to protect hamsters from acute infection with leptospires.

\section{MATERIALS AND METHODS}

Leptospires. Leptospira interrogans serovars pomona and hardjo were isolated from cases of human leptospirosis in the Waikato district of New Zealand (Christmas, Bragger and Till, 1974) and identified by the W.H.O. Leptospira Reference Laboratory, Brisbane, Australia, which also supplied other serovars used for extraction of F4 antigen (serovar-specific, lipopolysaccharide antigen), as described previously (Faine et al., 1974). Leptospires were cultured and counted as described by Adler and Faine (1976).

Isolation of Leptospires and collection of sera. A leptospirosis survey was organised in the Taranaki district of New Zealand (N.Z.) from September 1973 to March 1974. This is an intensive dairy-farming area that reports many cases of leptospirosis each spring and summer. Medical practitioners in the area were invited to participate by inoculating four bottles of EMJH medium - themodification by Johnson and Harris (1967) of the medium of Ellinghausen and McCullough - with the blood of patients suspected of having leptospirosis. The cultures were returned by post to the National Health Institute, Wellington, N.Z., where they were examined weekly for leptospires for a period of at least 2 months. Leptospires isolated from patients were subcultured, and were serotyped either at the W.H.O. Leptospira Reference Laboratory, Brisbane, Australia, or the National Health Institute. Subsequent specimens of serum were sent to the National Health Institute for leptospiral serology. Leptospires were isolated from 28 of a total of 101 patients. However, sera from some of the patients were not used for this research because later samples were not obtained. Several other patients from whom leptospires were not isolated subsequently developed leptospiral antibodies. If the infecting serovar could be identified fromantibodies in serum samples, the samples were included amongst those used for a comparison of the titres of MA and F4 antibodies (see Results and table IV); in all other parts of this investigation serum samples and data from patients from whom leptospires were isolated were used exclusively.

The serum samples from patients from whom serovar copenhageni was isolated were kindly provided by Dr C. Borg-Petersen, Statens Seruminstitut, Copenhagen, Denmark, who also identified the isolates.

Rabbit antisera were prepared by inoculating New Zealand White rabbits intravenously 
with $5 \mathrm{ml}$ of leptospiral culture grown in Korthof's medium with rabbit serum $10 \%$ (Alston and Broom, 1958).

Serological methods. The MA test was modified from Alston and Broom (1958) by the use of Cooke Microtitre equipment to prepare serum dilutions. The passive haemagglutination (HA) test for F4 antibodies was performed as described previously (Faine et al., 1974).

Serum was absorbed by resuspending approximately $10^{10}$ washed leptospires in a $0.1 \mathrm{ml}$ volume and incubating at $37^{\circ} \mathrm{C}$ for $90 \mathrm{~min}$.; the leptospires were then removed by centrifugation at $27000 \mathrm{~g}$ for $20 \mathrm{~min}$. It was necessary to absorb high-titre sera twice.

To absorb sera with $\mathrm{F} 4$ antigen, a volume of $0.2 \mathrm{ml}$ of a 1 in 4 dilution of serum inactivated at $56^{\circ} \mathrm{C}$ for $30 \mathrm{~min}$. was incubated at $37^{\circ} \mathrm{C}$ for $90 \mathrm{~min}$. with $0.5 \mathrm{ml}$ of packed $\mathrm{F} 4$ sensitised erythrocytes, which were then removed by centrifugation at $500 \mathrm{~g}$ for $10 \mathrm{~min}$.

Serum fractionation. Sucrose density gradients were prepared by sequentially layering $1 \cdot 1-\mathrm{ml}$ volumes of $40,30,20$ and $10 \%(\mathrm{w} / \mathrm{v})$ sucrose solutions in phosphate buffered saline

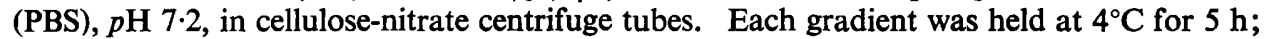
$0.25 \mathrm{ml}$ of serum was layered on top, and the sample was centrifuged for $18 \mathrm{~h}$ at 40000 r.p.m. $(157000 \mathrm{~g})$ and $4^{\circ} \mathrm{C}$ in a Beckman L265B ultracentrifuge. Ten fractions of $0.5 \mathrm{ml}$ were collected with a Beckman fraction-recovery system. The location of IgM and IgG was checked for each fractionated serum by means of immunodiffusion with specific anti-human IgM and IgG. IgM was found in fractions 2 and 3 and IgG in fractions 5 and 6 . The specific IgM and IgG antibody titres of a serum sample were taken to be four times higher than the recorded titres of pooled fractions 2 and 3 and pooled fractions 5 and 6 respectively; this was because the original serum was diluted $c .1$ in 4.

\section{TABLE I}

Homologous agglutinin and F4 antibody responses of patients infected with serovar pomona

\begin{tabular}{|c|c|c|c|}
\hline $\begin{array}{c}\text { Patient } \\
\text { no. }\end{array}$ & $\begin{array}{l}\text { Day of } \\
\text { illness }\end{array}$ & $\underset{\text { titre }}{\text { Agglutinin }}$ & $\underset{\text { titre }}{\text { F4 antibody }}$ \\
\hline 1 & $\begin{array}{r}16 \\
22 \\
33 \\
41 \\
115\end{array}$ & $\begin{array}{r}512 \\
8192 \\
16384 \\
2048 \\
256\end{array}$ & $\begin{array}{r}4096 \\
262144 \\
32768 \\
16384 \\
256\end{array}$ \\
\hline 2 & $\begin{array}{r}20 \\
41 \\
68 \\
110 \\
138 \\
154\end{array}$ & $\begin{array}{r}2048 \\
1024 \\
512 \\
256 \\
256 \\
256\end{array}$ & $\begin{array}{r}1024 \\
256 \\
64 \\
32 \\
32 \\
32\end{array}$ \\
\hline 3 & $\begin{array}{r}5 \\
11 \\
20 \\
49\end{array}$ & $\begin{array}{r}0 \\
2048 \\
8192 \\
2048\end{array}$ & $\begin{array}{r}0 \\
512 \\
1024 \\
64\end{array}$ \\
\hline 4 & $\begin{array}{r}3 \\
52 \\
73\end{array}$ & $\begin{array}{r}0 \\
512 \\
512\end{array}$ & $\begin{array}{r}0 \\
1024 \\
1024\end{array}$ \\
\hline 5 & $\begin{array}{r}2 \\
8 \\
13 \\
278\end{array}$ & $\begin{array}{r}0 \\
0 \\
2048 \\
128\end{array}$ & $\begin{array}{r}0 \\
32 \\
2048 \\
256\end{array}$ \\
\hline
\end{tabular}

\begin{tabular}{|c|c|c|c|}
\hline $\begin{array}{l}\text { Patient } \\
\text { no. }\end{array}$ & $\begin{array}{l}\text { Day of } \\
\text { illness }\end{array}$ & $\underset{\text { titre }}{\text { Agglutinin }}$ & $\underset{\text { titre }}{\mathrm{F} 4}$ antibody \\
\hline 6 & $\begin{array}{r}2 \\
42 \\
107 \\
113 \\
230\end{array}$ & $\begin{array}{r}0 \\
256 \\
512 \\
512 \\
256\end{array}$ & $\begin{array}{r}0 \\
2048 \\
512 \\
512 \\
256\end{array}$ \\
\hline 7 & $\begin{array}{r}4 \\
36\end{array}$ & $\begin{array}{r}0 \\
256\end{array}$ & $\begin{array}{r}0 \\
2048\end{array}$ \\
\hline 8 & $\begin{array}{r}1 \\
87\end{array}$ & $\begin{array}{r}0 \\
128\end{array}$ & $\begin{array}{r}0 \\
64\end{array}$ \\
\hline 9 & $\begin{array}{r}2 \\
14 \\
165\end{array}$ & $\begin{array}{r}0 \\
128 \\
128\end{array}$ & $\begin{array}{r}0 \\
256 \\
32\end{array}$ \\
\hline 10 & $\begin{array}{r}2 \\
15 \\
20 \\
138\end{array}$ & $\begin{array}{r}0 \\
2048 \\
1024 \\
256\end{array}$ & $\begin{array}{r}0 \\
128 \\
256 \\
64\end{array}$ \\
\hline 11 & $\begin{array}{r}13 \\
22 \\
38 \\
50 \\
129\end{array}$ & $\begin{array}{r}0 \\
512 \\
256 \\
256 \\
16\end{array}$ & $\begin{array}{r}0 \\
32 \\
16 \\
8 \\
8\end{array}$ \\
\hline
\end{tabular}

Agglutinin and F4 antibody titres were measured by the MA and HA tests respectively. 
TABLE II

Homologous agglutinin and F4 antibody responses of patients infected with serovar hardjo

\begin{tabular}{|c|c|c|c|c|c|c|c|}
\hline $\begin{array}{c}\text { Patient } \\
\text { no. }\end{array}$ & $\begin{array}{l}\text { Day of } \\
\text { illness }\end{array}$ & $\underset{\text { titre }}{\text { Agglutinin }}$ & $\underset{\text { titre }}{\text { F4 antibody }}$ & $\begin{array}{l}\text { Patient } \\
\text { no. }\end{array}$ & $\begin{array}{l}\text { Day of } \\
\text { illness }\end{array}$ & $\underset{\text { titre }}{\text { Agglutinin }}$ & $\underset{\text { titre }}{\text { F4 antibody }}$ \\
\hline \multirow[t]{2}{*}{12} & $\begin{array}{r}7 \\
25 \\
38 \\
44\end{array}$ & $\begin{array}{l}1024 \\
2048 \\
2048 \\
1024\end{array}$ & $\begin{array}{l}8192 \\
8192 \\
8192 \\
2048\end{array}$ & 17 & $\begin{array}{r}4 \\
26 \\
120\end{array}$ & $\begin{array}{r}0 \\
32 \\
2048\end{array}$ & $\begin{array}{r}0 \\
2048 \\
128\end{array}$ \\
\hline & $\begin{array}{l}77 \\
82\end{array}$ & $\begin{array}{l}256 \\
256\end{array}$ & $\begin{array}{l}2048 \\
2048\end{array}$ & \multirow[t]{2}{*}{18} & $\begin{array}{r}73 \\
113 \\
215\end{array}$ & $\begin{array}{r}1024 \\
1024 \\
512\end{array}$ & $\begin{array}{r}4096 \\
4096 \\
512\end{array}$ \\
\hline \multirow[t]{2}{*}{13} & $\begin{array}{r}3 \\
88\end{array}$ & $\begin{array}{r}0 \\
256\end{array}$ & $\begin{array}{r}32 \\
256\end{array}$ & & 302 & 512 & 512 \\
\hline & $\begin{array}{l}121 \\
300\end{array}$ & $\begin{array}{l}128 \\
128\end{array}$ & $\begin{array}{r}128 \\
64\end{array}$ & 19 & $\begin{array}{r}1 \\
6 \\
42\end{array}$ & $\begin{array}{r}0 \\
128 \\
512\end{array}$ & $\begin{array}{r}0 \\
32 \\
128\end{array}$ \\
\hline 14 & $\begin{array}{r}1 \\
26 \\
63 \\
90\end{array}$ & $\begin{array}{r}0 \\
4096 \\
2048 \\
2048\end{array}$ & $\begin{array}{r}0 \\
512 \\
128 \\
64\end{array}$ & 20 & $\begin{array}{r}4 \\
8 \\
24\end{array}$ & $\begin{array}{r}0 \\
1024 \\
1024\end{array}$ & $\begin{array}{r}0 \\
128 \\
128\end{array}$ \\
\hline 15 & $\begin{array}{r}4 \\
28 \\
63\end{array}$ & $\begin{array}{r}0 \\
4096 \\
2048\end{array}$ & $\begin{array}{r}0 \\
1024 \\
1024\end{array}$ & \multirow[t]{2}{*}{21} & $\begin{array}{r}1 \\
13 \\
219\end{array}$ & $\begin{array}{r}0 \\
2048 \\
128\end{array}$ & $\begin{array}{r}0 \\
16384 \\
64\end{array}$ \\
\hline 16 & $\begin{array}{r}5 \\
18 \\
31\end{array}$ & $\begin{array}{r}0 \\
1024 \\
1024\end{array}$ & $\begin{array}{r}0 \\
128 \\
64\end{array}$ & & & & \\
\hline
\end{tabular}

Footnote as in table I.

TABLE III

Homologous agglutinin and F4 antibody responses of patients infected with serovars ballum and copenhageni

\begin{tabular}{|c|c|c|c|c|c|c|c|}
\hline $\begin{array}{l}\text { Patient } \\
\text { no. }\end{array}$ & $\begin{array}{l}\text { Day of } \\
\text { illness }\end{array}$ & $\underset{\text { titre }}{\text { Agglutinin }}$ & $\underset{\text { titre }}{\text { F4 antibody }}$ & $\begin{array}{c}\text { Patient } \\
\text { no. }\end{array}$ & $\begin{array}{l}\text { Day of } \\
\text { illness }\end{array}$ & $\underset{\text { titre }}{\text { Agglutinin }}$ & $\underset{\text { titre }}{\text { F4 antibody }}$ \\
\hline $22^{*}$ & $\begin{array}{r}8 \\
13\end{array}$ & $\begin{array}{l}256 \\
256\end{array}$ & $\begin{array}{r}64 \\
128\end{array}$ & $26 \dagger$ & $\begin{array}{r}7 \\
25 \\
35\end{array}$ & $\begin{array}{r}0 \\
1024 \\
1024\end{array}$ & $\begin{array}{l}128 \\
512 \\
512\end{array}$ \\
\hline $23^{*}$ & $\begin{array}{r}2 \\
9 \\
21 \\
28\end{array}$ & $\begin{array}{r}0 \\
0 \\
1024 \\
1024\end{array}$ & $\begin{array}{r}0 \\
0 \\
32 \\
32\end{array}$ & $27 \dagger$ & $\begin{array}{r}6 \\
11 \\
27\end{array}$ & $\begin{array}{r}0 \\
256 \\
8192\end{array}$ & $\begin{array}{r}256 \\
4096 \\
4096\end{array}$ \\
\hline $24 \dagger$ & $\begin{array}{r}6 \\
13 \\
33\end{array}$ & $\begin{array}{r}256 \\
2048 \\
2048\end{array}$ & $\begin{array}{r}1024 \\
32768 \\
16384\end{array}$ & $28 \dagger$ & $\begin{array}{r}9 \\
37\end{array}$ & $\begin{array}{r}0 \\
1024\end{array}$ & $\begin{array}{l}256 \\
256\end{array}$ \\
\hline $25 \dagger$ & $\begin{array}{r}7 \\
14 \\
30\end{array}$ & $\begin{array}{r}128 \\
1024 \\
8192\end{array}$ & $\begin{array}{r}512 \\
2048 \\
2048\end{array}$ & & & & \\
\hline
\end{tabular}

Footnote as in table I. * Serovar ballum isolated. † Serovar copenhageni isolated. 
Hamster protection test. Golden hamsters aged 4-6 weeks and bred and housed at the National Health Institute were used. Two hamsters were inoculated intraperitoneally with each antiserum dilution in a volume of $0.1 \mathrm{ml}$, unless otherwise stated, and challenged $4 \mathrm{~h}$ later by the same route with at least $2 \times 10^{8}$ serovar pomona in $1.0 \mathrm{ml}$ of EMJH medium. Serum was sterilised by filtration through a $0.22 \mu \mathrm{m}$ Millipore membrane filter. Control hamsters always died within 3 or 4 days of inoculation, while protected animals survived for at least 14 days.

\section{RESULTS}

The MA and F4 antibody response of human patients infected with leptospires

Tables I-III show the antibody responses of patients with leptospirosis that had been proved by isolation of the infecting serovar (pomona, hardjo, ballum or copenhageni) from the bloodstream during the acute febrile stage of illness. All patients developed homologous agglutinating antibody and F4 antibody. In some patients (e.g. nos. 11 and 23), agglutinin titres were higher than F4 antibody titres, while in others (e.g. nos. 1 and 24) the titres of F4 antibody exceeded those of agglutinin. In general the levels of the two types of antibody rose and fell at approximately the same time. Serum samples taken from 18 patients at a time when leptospires were shown by cultural methods to be present in the bloodstream contained neither agglutinating nor F4 antibodies. However, five sera taken early in infection (patients 5, 13, 26, 27 and 28) contained demonstrable levels of F4 but not agglutinating antibody, suggesting that the former appears earlier than the latter. In two other patients F4 antibody reached a peak earlier than did agglutinin (patients 1 and 17).

TABLE IV

Relationship between the titres of agglutinating and F4 antibodies against the infecting serovar* in the sera of 220 leptospirosis patients

\begin{tabular}{|c|c|c|c|c|c|c|c|c|c|c|c|c|c|c|c|}
\hline \multirow{2}{*}{$\begin{array}{c}\text { Agglutinin } \\
\text { titres in } \\
\text { patients' } \\
\text { serum }\end{array}$} & \multicolumn{15}{|c|}{ Number of patients whose serum contained F 4 antibody at a titre of } \\
\hline & 0 & $N$ & $\theta$ & $\infty$ & 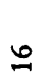 & లె & t & $\stackrel{\infty}{\cong}$ & 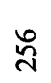 & $\frac{N}{n}$ & ఫ్ & 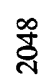 & ஓ̊ & 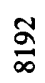 & $\stackrel{a}{\infty}$ \\
\hline 0 & 49 & - & - & 1 & 1 & 1 & 2 & - & - & 1 & - & - & - & - & - \\
\hline 2 & - & - & - & - & - & - & - & - & - & - & - & - & - & - & - \\
\hline 4 & - & - & - & $\begin{array}{lll}- & \\
-1\end{array}$ & - & - & - & - & - & - & - & - & - & - & - \\
\hline 8 & - & - & - & $\begin{array}{ll}- & \text { r }\end{array}$ & - & - & - & - & - & - & - & - & - & - & - \\
\hline 16 & - & - & $=$ & - & - & - & - & - & $=$ & $=$ & $=$ & 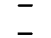 & $\overline{-}$ & $\overline{-}$ & $=$ \\
\hline $\begin{array}{l}32 \\
64\end{array}$ & $\overline{-}$ & $\overline{-}$ & $=$ & $\overline{2}$ & $\overline{1}$ & $\overline{4}$ & $\overline{6}$ & $\overline{1}$ & $\overline{1}$ & - & $=$ & - & - & - & - \\
\hline 128 & - & - & - & - & 2 & 10 & 6 & 7 & 2 & 1 & - & 3 & - & - & - \\
\hline 256 & - & - & - & - & 4 & 4 & 8 & 4 & 9 & 3 & - & 1 & - & 1 & - \\
\hline 512 & - & - & - & - & - & 2 & 1 & 4 & 4 & 3 & 5 & 1 & 1 & - & - \\
\hline 1024 & - & - & - & 1 & 1 & 2 & 3 & 6 & 5 & 4 & 2 & 4 & 2 & 2 & 3 \\
\hline 2048 & - & - & - & - & - & - & - & 2 & 2 & 2 & - & 4 & 3 & 4 & 1 \\
\hline 4096 & - & - & - & - & - & - & - & - & 2 & 3 & 3 & - & - & - & - \\
\hline 8192 & - & - & - & - & - & - & - & - & 1 & 1 & - & - & 1 & - & 1 \\
\hline$>8192$ & - & - & - & - & - & - & - & - & - & - & - & - & - & - & 1 \\
\hline
\end{tabular}

* Serovar either isolated or established serologically.

Agglutinin and F4 antibody titres were measured by the MA and HA tests respectively. 
Homologous F4 antibody was found in sera from all patients with leptospirosis, whether the disease was diagnosed by culture or serologically by demonstrating $a \geqslant 4$-fold rise in agglutinin titre in paired sera. The combined results of all sera are shown in table IV. In none of 49 MA test-negative sera was F4 antibody detected. The six patients whose sera were initially HA positive and MA negative all developed agglutinin titres later.

\section{Classes of antibody involved in the human immune response}

Human sera from 14 of the patients in tables I-III were fractionated on sucrose density gradients and the IgM and IgG fractions tested serologically by the MA and HA tests. The F4 antibodies were invariably restricted to the IgM class, even as late as 10 months after infection. In contrast, agglutinins were found initially in the IgM fraction only, but in some patients from whom later serum samples were available, IgG agglutinins were produced during the convalescent stage of illness several weeks after infection (table V). The other

TABLE V

Antibody response of the five patients (of 14 tested) who produced both IgM and IgG agglutinins

\begin{tabular}{|c|c|c|c|c|c|}
\hline \multirow{2}{*}{$\begin{array}{l}\text { Patient } \\
\text { no. }\end{array}$} & \multirow{2}{*}{$\begin{array}{l}\text { Serovar } \\
\text { isolated }\end{array}$} & \multirow{2}{*}{$\begin{array}{l}\text { Day of } \\
\text { illness }\end{array}$} & \multicolumn{2}{|c|}{$\begin{array}{l}\text { Agglutinin titre in the } \\
\text { immunoglobulin class }\end{array}$} & \multirow{2}{*}{$\begin{array}{l}\text { F4 antibody } \\
\text { titre in } \\
\text { the immuno- } \\
\text { globulin } \\
\text { class IgM* }\end{array}$} \\
\hline & & & IgM & IgG & \\
\hline 1 & pomona & $\begin{array}{r}16 \\
22 \\
33 \\
41 \\
115\end{array}$ & $\begin{array}{r}512 \\
8192 \\
16384 \\
2048 \\
256\end{array}$ & $\begin{array}{r}\mathbf{0} \\
\mathbf{0} \\
\mathbf{0} \\
\mathbf{0} \\
128\end{array}$ & $\begin{array}{r}4096 \\
262144 \\
32768 \\
16384 \\
256\end{array}$ \\
\hline 2 & pomona & $\begin{array}{r}20 \\
41 \\
68 \\
110 \\
138 \\
154\end{array}$ & $\begin{array}{r}2048 \\
1024 \\
512 \\
256 \\
128 \\
128\end{array}$ & $\begin{array}{r}0 \\
0 \\
4 \\
32 \\
64 \\
32\end{array}$ & $\begin{array}{r}1024 \\
256 \\
64 \\
32 \\
32 \\
32\end{array}$ \\
\hline 5 & pomona & $\begin{array}{r}2 \\
8 \\
13 \\
278\end{array}$ & $\begin{array}{r}0 \\
0 \\
1024 \\
64\end{array}$ & $\begin{array}{r}0 \\
0 \\
0 \\
128\end{array}$ & $\begin{array}{r}0 \\
32 \\
2048 \\
256\end{array}$ \\
\hline 6 & pomona & $\begin{array}{r}2 \\
42 \\
107 \\
113 \\
230\end{array}$ & $\begin{array}{r}0 \\
256 \\
512 \\
512 \\
32\end{array}$ & $\begin{array}{r}0 \\
32 \\
128 \\
128 \\
128\end{array}$ & $\begin{array}{r}0 \\
2048 \\
512 \\
512 \\
256\end{array}$ \\
\hline 14 & hardjo & $\begin{array}{l}1 \\
26 \\
63 \\
90\end{array}$ & $\begin{array}{r}0 \\
4096 \\
2048 \\
2048\end{array}$ & $\begin{array}{r}0 \\
0 \\
32 \\
64\end{array}$ & $\begin{array}{r}0 \\
512 \\
128 \\
64\end{array}$ \\
\hline
\end{tabular}

* F4 antibodies (measured by HA) were found only in the IgM fraction.

Agglutinins were measured by the MA test. 
TABLE VI

Absorption of human sera, from cases of serovar pomona infection, with homologous leptospires or with F4

\begin{tabular}{|c|c|c|c|c|c|c|c|}
\hline \multirow{2}{*}{$\begin{array}{l}\text { Patient } \\
\text { no. }\end{array}$} & \multirow{2}{*}{$\begin{array}{l}\text { Day of } \\
\text { illness on } \\
\text { which } \\
\text { serum was } \\
\text { taken }\end{array}$} & \multicolumn{2}{|c|}{ Titre of } & \multicolumn{2}{|c|}{$\begin{array}{c}\begin{array}{c}\text { Titre after } \\
\text { absorption with } \\
\text { leptospires }\end{array} \\
\text {. }\end{array}$} & \multicolumn{2}{|c|}{$\begin{array}{c}\begin{array}{c}\text { Titre after } \\
\text { absorption with } \\
\text { F4 antigen }\end{array}\end{array}$} \\
\hline & & agglutinin & F4 antibody & Ágglutinin & F4 antibody & Ágglutinin & F4 antibody \\
\hline $\begin{array}{l}1 \\
1 \\
1 \\
4 \\
6\end{array}$ & $\begin{array}{r}16 \\
41 \\
115 \\
73 \\
113\end{array}$ & $\begin{array}{r}512 \\
2048 \\
256 \\
512 \\
512\end{array}$ & $\begin{array}{r}4096 \\
16384 \\
256 \\
1024 \\
512\end{array}$ & $\begin{array}{r}0 \\
16 \\
0 \\
2 \\
0\end{array}$ & $\begin{array}{r}2 \\
16 \\
0 \\
4 \\
0\end{array}$ & $\begin{array}{r}256 \\
1024 \\
256 \\
256 \\
512\end{array}$ & $\begin{array}{r}8 \\
32 \\
0 \\
8 \\
4\end{array}$ \\
\hline
\end{tabular}

Agglutinin and F4 antibody titres were measured by the MA and HA tests respectively.

\section{TABLE VII}

Cross-reactions of human and rabbit immune sera with F4 antigen from heterologous leptospiral serovars

\begin{tabular}{|c|c|c|c|c|c|c|c|c|c|c|c|c|c|c|c|}
\hline \multirow{3}{*}{$\begin{array}{c}\text { Heterologous } \\
\text { serovars from } \\
\text { which F4 } \\
\text { antigen } \\
\text { obtained }\end{array}$} & \multicolumn{15}{|c|}{$\begin{array}{l}\text { F4 antibody titres }(\log 2) \text { in patients }(A-K) \text { infected, or rabbits (R1-R4) } \\
\text { immunised, with serovars }\end{array}$} \\
\hline & \multicolumn{3}{|c|}{ copenhageni } & \multicolumn{3}{|c|}{ ballum } & \multicolumn{6}{|c|}{ pomona } & \multicolumn{3}{|c|}{ hardjo } \\
\hline & A & B & $\overrightarrow{\mathrm{R} 1}$ & $\mathrm{C}$ & D & $\mathrm{R} 2$ & E & $\mathbf{F}$ & $G$ & $\mathbf{H}$ & I & R3 & J & $\mathbf{K}$ & R4 \\
\hline$R G A$ & 2 & 7 & 11 & 3 & 2 & - & 4 & 5 & 7 & 9 & 9 & 9 & 8 & 6 & - \\
\hline copenhageni & $\overline{9}$ & 12 & 11 & 5 & 4 & 4 & 5 & 7 & 8 & 10 & 10 & 8 & 8 & 9 & 9 \\
\hline canicola & - & 6 & 7 & 6 & 4 & - & 4 & 7 & 6 & - & - & 6 & 8 & 6 & - \\
\hline schüffneri & 4 & 7 & 8 & 5 & 4 & - & 3 & 4 & 6 & - & 5 & 7 & 8 & 6 & - \\
\hline ballum & NT & NT & - & NT & NT & 8 & NT & NT & 7 & 5 & - & - & NT & NT & 7 \\
\hline castellonis & 11 & 14 & - & 8 & 8 & 11 & 7 & 10 & 9 & 11 & 10 & $\bar{z}$ & 10 & 11 & 8 \\
\hline zanoni & 6 & 10 & 8 & 5 & 4 & - & 5 & 8 & 6 & 9 & 9 & 7 & 9 & 8 & - \\
\hline robinsoni & 13 & 15 & 4 & 11 & 8 & - & 9 & 13 & 9 & 12 & 12 & 9 & 13 & 13 & 6 \\
\hline cynopteri & NT & NT & - & NT & NT & - & NT & NT & $=$ & $\overline{7}$ & $\bar{z}$ & $=$ & NT & NT & 5 \\
\hline butembo & 6 & 10 & - & 6 & 5 & - & 4 & 7 & 7 & 7 & 7 & 5 & 9 & 8 & 5 \\
\hline autumnalis & - & 4 & $\overline{-}$ & 4 & - & - & 3 & 6 & - & - & - & 3 & 9 & 5 & - \\
\hline rachmati & $=$ & 5 & 7 & 6 & 4 & - & 6 & 5 & - & - & - & 9 & 9 & 5 & - \\
\hline djasiman & 7 & 10 & 6 & 4 & 4 & - & 4 & 6 & - & - & - & 5 & 8 & 8 & - \\
\hline australis & 4 & 6 & - & 5 & 6 & - & 4 & 6 & - & 12 & 12 & 6 & 10 & 6 & 8 \\
\hline bratislava & - & - & - & - & - & - & $\overline{\bar{z}}$ & 2 & $\overline{0}$ & - & 6 & 8 & - & - & - \\
\hline pomona & 10 & 13 & 6 & 6 & 5 & - & 7 & 10 & 9 & 12 & 11 & 10 & 11 & 9 & 4 \\
\hline grippotyphosa & - & $\overline{\mathrm{a}}$ & - & - & $\overline{-}$ & - & - & $\bar{a}$ & $\bar{z}$ & $\bar{z}$ & - & - & $\overline{\overline{7}}$ & - & $\bar{c}$ \\
\hline kremastos & $\overline{0}$ & 8 & - & 2 & 5 & 4 & $=$ & 3 & 6 & 7 & - & - & 7 & 9 & 6 \\
\hline hardjo & 10 & 11 & 6 & - & 2 & - & 6 & 5 & 6 & - & - & 4 & 5 & 4 & 10 \\
\hline medanensis & 9 & 13 & - & 7 & 6 & - & 6 & 9 & 9 & 10 & 9 & $=$ & 11 & 11 & 9 \\
\hline wolffi & 8 & 13 & - & 6 & 7 & 4 & 6 & 9 & 7 & 8 & 7 & 6 & 10 & 11 & 6 \\
\hline patoc & 13 & 16 & - & 9 & 8 & - & 9 & 10 & 9 & 12 & 12 & - & 13 & 12 & 8 \\
\hline
\end{tabular}

RGA = Reference strain of serovar icterohaemorrhagiae (Icterohaemorrhagiae group).

Patients A, B, C, D, E, F, G, H, and I are referred to in other tables as patients $25,24,22,23,10,4,6,1$ and 3 respectively.

- = Nil; NT $=$ not tested as supply of material was exhausted. 
patients tested produced only IgM agglutinins (patients $3,4,12,13,24,25,26$, 27 and 28).

\section{Relationship between F4 and agglutinating antibodies}

Tables I and III show that certain sera contained F4 antibodies but no agglutinins; the work of Adler and Faine (1978), which showed that rabbit F4 antibody did not agglutinate leptospires, may be pertinent. The results of absorption tests with sera from five patients infected with serovar pomona (table VI) show that leptospires absorbed both F4 antibody and agglutinin, while F4-sensitised erythrocytes removed only F4 antibody, without significantly reducing the agglutinin titre.
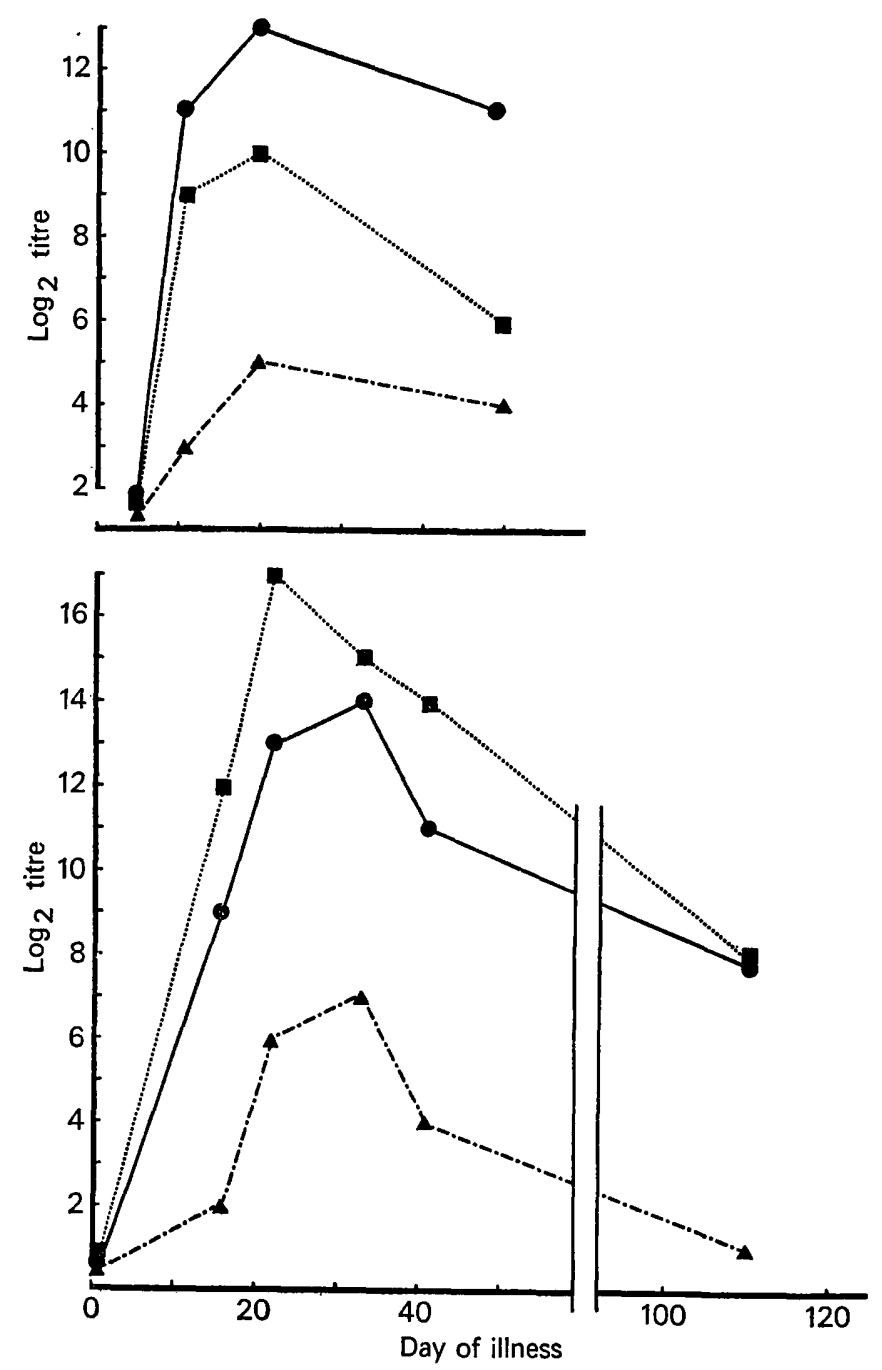

FIG. 1. Agglutinin ( titres of sera taken at various times after onset of illness from patient no. 2 (above) and patient no. 1 (below). 


\section{Reaction of human sera with heterologous F4 antigen}

The reactions of selected sera from patients infected with different leptospiral serovars are shown in table VII and compared with the reactions of rabbit antisera. The human sera cross-reacted widely with F4 antigen from heterologous serovars. The cross-reactions among patients infected with the same serovar were inconsistent, and there was no correlation between the crossreactions of human and rabbit sera. F4 from certain serovars (e.g. robinsoni) cross-reacted strongly with human sera, while F4 from others (e.g. grippotyphosa) did not cross-react at all. The titre against serovar patoc (Biflexa complex) F4 was at least as high as the homologous titre and sometimes considerably higher.
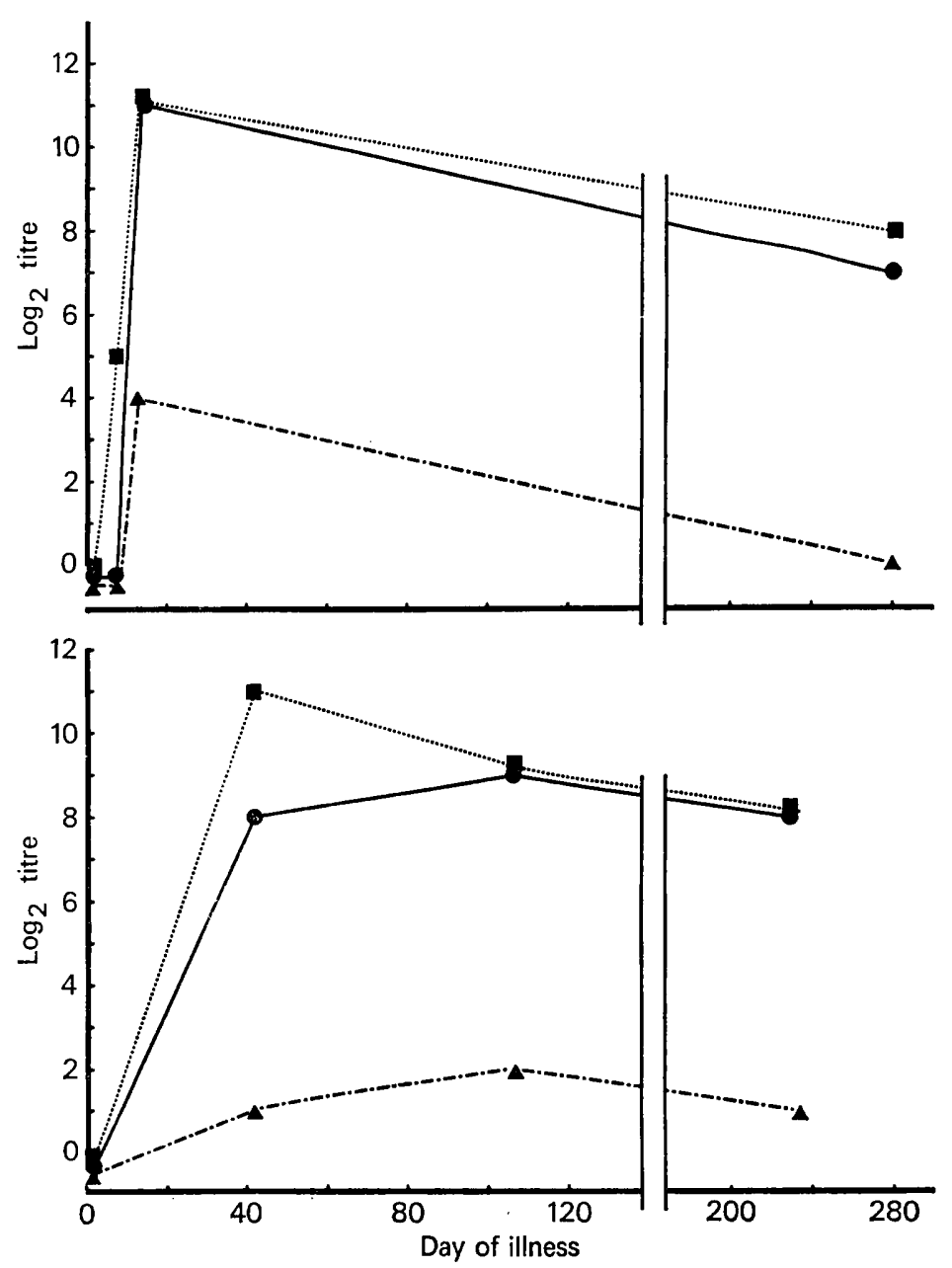

FIG. 2. Agglutinin $(\bullet-\bullet)$, F4 antibody $(\boldsymbol{\square} \cdots \boldsymbol{\square})$ and hamster-protective $(\boldsymbol{\Delta}-\cdot-\cdot-\boldsymbol{\Delta})$ titres of sera taken at various times after onset of illness from patient no. 5 (above) and patient no. 6 (below). 


\section{Hamster-protection tests with human sera}

Patients infected with serovar pomona, and from whom several serum samples were available, were examined in respect of the ability of their serum to protect hamsters from acute infection with the same organism. Figs. 1 and 2 show that the capacity to protect hamsters correlated well with the agglutinin titre; correlation with the F4 antibody titre was less close.

In order to compare the protective capacities of antibodies in the $\operatorname{IgM}$ and IgG classes, appropriate sucrose density-gradient fractions were titrated in hamster protection tests. The results (table VIII) showed that both IgM and IgG fractions protected hamsters from lethal infections with serovar pomona. The protective capacity of either fraction correlated well with its level of agglutinating antibody.

Sera from patients infected with serovars hardjo, ballum or copenhageni were tested for their capacity to protect hamsters from infection with serovar pomona. These heterologous sera protected only if they possessed crossreacting agglutinins to serovar pomona. Heterologous sera with antibodies reacting with the $\mathrm{F} 4$ antigen of serovar pomona did not protect unless they also had cross-reacting agglutinins (table IX).

\section{Discussion}

In human leptospirosis, it is impossible to draw firm conclusions about the homologous antibody response unless the infecting serovar has been isolated and identified (Borg-Petersen, 1953). In 10\% of patients Combiescu et al. (1960) could not identify the infecting serovar by the antibody response, even when repeated serum samples were taken. Earlier workers (Pike et al., 1965; Tong et al., 1971; Sulzer et al., 1975) either failed to isolate and identify the infecting serovar, or examined sera only in the early stages of illness, or gave insufficient information.

\section{TABLE VIII}

Capacity of IgM and IgG fractions of immune sera from patients infected with serovar pomona to protect hamsters from infection with $2 \times 10^{8}$ homologous leptospires

\begin{tabular}{|c|c|c|c|c|c|}
\hline $\begin{array}{l}\text { Patient } \\
\text { no. }\end{array}$ & $\begin{array}{l}\text { Day of } \\
\text { illness on } \\
\text { which } \\
\text { serum taken }\end{array}$ & Fraction & $\underset{\text { titre }}{\text { Agglutinin }}$ & $\underset{\text { titre }}{\text { F4 antibody }}$ & $\begin{array}{c}\text { Hamster } \\
\text { protective } \\
\text { titre* }\end{array}$ \\
\hline $\begin{array}{l}1 \\
1\end{array}$ & $\begin{array}{l}115 \\
115\end{array}$ & $\begin{array}{l}\text { IgM } \\
\text { IgG }\end{array}$ & $\begin{array}{l}256 \\
128\end{array}$ & $\begin{array}{r}256 \\
0\end{array}$ & $\begin{array}{l}4 \\
2\end{array}$ \\
\hline $\begin{array}{l}5 \\
5\end{array}$ & $\begin{array}{l}278 \\
278\end{array}$ & $\begin{array}{l}\text { IgM } \\
\text { IgG }\end{array}$ & $\begin{array}{r}64 \\
128\end{array}$ & $\begin{array}{r}256 \\
0\end{array}$ & $\frac{1}{2}$ \\
\hline $\begin{array}{l}6 \\
6\end{array}$ & $\begin{array}{l}113 \\
113\end{array}$ & $\begin{array}{l}\text { IgM } \\
\text { IgG }\end{array}$ & $\begin{array}{l}512 \\
128\end{array}$ & $\begin{array}{r}512 \\
0\end{array}$ & $\begin{array}{l}8 \\
2\end{array}$ \\
\hline
\end{tabular}

* The dose of serum, or serum dilution, was $0.2 \mathrm{ml}$ per hamster.

Agglutinin and F4 antibody titres were measured by the MA and HA tests respectively. 
TABLE IX

Protective capacity of immune sera from patients infected with leptospiral serovars other than pomona, for hamsters challenged with pomona $\left(2 \times 10^{8}\right.$ organisms $)$

\begin{tabular}{|c|c|c|c|c|c|}
\hline \multirow{2}{*}{$\begin{array}{l}\text { Serovar with } \\
\text { which patient } \\
\text { was infected }\end{array}$} & \multicolumn{2}{|c|}{$\underset{\text { titre }}{\text { Homologous antibody }}$} & \multicolumn{2}{|c|}{$\begin{array}{l}\text { Antibody titre against } \\
\text { serovar pomona }\end{array}$} & \multirow{2}{*}{$\underset{\text { protection }}{\text { Hamster }}$} \\
\hline & Aggultinin & F4 antibody & Agglutinin & F4 antibody & \\
\hline $\begin{array}{l}\text { hardjo } \\
\text { ballum } \\
\text { copenhageni }\end{array}$ & $\begin{array}{l}2048 \\
1024 \\
1024\end{array}$ & $\begin{array}{r}16384 \\
32 \\
2048\end{array}$ & $\begin{array}{l}0 \\
0 \\
0\end{array}$ & $\begin{array}{r}4096 \\
32 \\
1024\end{array}$ & $\begin{array}{l}\bar{z} \\
-\end{array}$ \\
\hline $\begin{array}{l}\text { hardjo } \\
\text { hardjo } \\
\text { hardjo }\end{array}$ & $\begin{array}{l}2048 \\
1024 \\
4096\end{array}$ & $\begin{array}{r}512 \\
16384 \\
8192\end{array}$ & $\begin{array}{r}1024 \\
128 \\
256\end{array}$ & $\begin{array}{r}512 \\
8192 \\
8192\end{array}$ & $\begin{array}{l}+ \\
+ \\
+\end{array}$ \\
\hline
\end{tabular}

* The dose of serum per hamster was $0.2 \mathrm{ml}$.

$t=$ Protection; $-=$ no protection.

No sera with agglutinins to serovar pomona were available from patients with ballum or copenhageni infections.

The results show (table V) that patients infected with leptospires can produce IgG agglutinins, but not all patients did so (e.g., patient no. 13). It was not possible to determine the frequency with which IgG agglutinins were produced because their time of appearance varied considerably, and serum samples were sometimes taken only in the early stages of infection. Antibiotic treatment early in the course of illness may have affected the production of IgG, as such treatment can suppress or delay the development of agglutinins (Babudieri, 1961). The IgM response was higher than the IgG response and persisted for many months regardless of IgG production. Rabbits, on the other hand, were found by Graves and Faine (1970) to produce high levels of IgG as well as IgM agglutinins. Moreover, both IgM and IgG classes of antibody contributed to the agglutinating activity of immune cattle sera (Morris and Hussaini, 1974). In immune guinea-pig sera, IgM was the predominant agglutinin until the 19th day of infection (Crawford, 1972), when IgG agglutinins appeared and soon predominated. Thus, unlike man, laboratory and domestic animals appear to follow the classical IgM and IgG pattern in their antileptospiral agglutinin response.

Patients infected with one of the four serovars examined always produced F4 antibody as well as agglutinin (tables I-III.) The titres did not necessarily correlate, but the times of appearance and disappearance of the two types of antibody were similar, although F4 antibodies sometimes appeared earlier than agglutinins. Unlike agglutinins, F4 antibodies were confined to the IgM class, a finding characteristic of antibodies to polysaccharide-type antigens (Pike, 1967).

The F4 antibodies appeared to be distinct from the agglutinins as shown by absorption studies (table VI), in which F4 antibodies contributed slightly, if at all, to the agglutinating titres of human sera. The fact that some sera contained F4 antibody but no agglutinin (tables I and III) suggested that F4 antibodies did 
not agglutinate leptospires. Adler and Faine (1978) found that F4 antibodies in immune rabbit sera combined with, but did not agglutinate, leptospires. It was not established whether human F4 antibodies also combined with leptospires. Antibodies against HL antigen were shown by McComb et al. (1957) to be distinct from agglutinins.

Human sera cross-reacted widely with heterologous F4 antigen (table VII), including that from serovar patoc (Biflexa complex); rabbit antisera, on the other hand, have been found to cross-react very little with F4 antigen from serovar patoc (Adler and Faine, unpublished observations). HL and F4 antigens were differentiated serologically on the basis of their reactions with rabbit antisera (Faine et al., 1974). It is unlikely that the cross-reaction of human sera was due to genus-specific components in the F4 antigen, because the sera did not react at all with $\mathrm{F} 4$ from certain serovars. The F4 antigen may contain a common backbone structure that is exposed to different degrees in different serovars; this would explain the inconsistent cross-reactivity of both human and rabbit sera, but there is insufficient information about the structure of either F4 or HL antigens to speculate further. Changes in cross-reactivity of F4 antibody during different stages of illness were not investigated, but an increase in specificity with time seems unlikely in view of the exclusively IgM nature of the anti-F4 response in man.

The protective capacity of human sera correlated well with agglutinin titre, and both IgM and IgG fractions protected hamsters (table VIII). Negi, Myers and Segre (1971) reported a similar finding with the sera of vaccinated calves, in which both IgM and IgG agglutinins protected hamsters from acute infection with serovar pomona. It seems that neither $\mathrm{F} 4$ nor $\mathrm{HL}$ antibodies are necessary for protection, as human IgG fractions contained neither. The protective capacity of serum appeared to be correlated with agglutinin, because hamsters were protected from serovar pomona infection by human heterologous agglutinin only if the sera possessed agglutinins against serovar pomona (table IX). Heterologous sera that reacted with F4 antigen of serovar pomona did not protect hamsters from pomona infection unless the sera also possessed agglutinins for serovar pomona; furthermore, absorption of sera with whole leptospires removed both the protective capacity and agglutinins, but absorption with F4 antigen had no significant effect on either.

Reports on the relationship between agglutinin titres and the ability of animal sera to protect hamsters are conflicting. Morsi, Shibley and Strother (1973) found that maximal agglutinin and protective antibody titres occurred at different times in swine after vaccination and after subsequent challenge. Negi et al. (1971) stated that 12 months after calves were vaccinated against pomona they had protective antibodies but no agglutinating antibodies; however, the initial serum dilutions used (1 in 100) in the MA test would not have detected low levels of agglutinins. Their findings that both IgM and IgG antibodies protected hamsters was similar to that obtained with human sera in the present study. Johnson, Bey and Auran (1975) found that the agglutinin titres in serum samples from cows vaccinated with a preparation of the outer envelope of serovar pomona correlated well with the ability of the samples to 
protect hamsters from infection with homologous leptospires. Our findings showed that the ability of human sera to protect hamsters from acute infection depended on the agglutinin titres. Similar results were obtained with immune rabbit sera (Adler and Faine, 1978).

The findings reported are significant in relation to immunity in human populations and in individuals. As long as the relevant agglutinins persist, it may be predicted that immunity to homologous and to certain heterologous leptospiral serovars will remain. Before undertaking an immunisation programme, it is essential to isolate and identify the infecting serovars.

\section{SUMMARY}

Antibody responses were studied in human patients from whom leptospiral serovars-mainly pomona or hardjo-had been isolated and identified. The antibody to the polysaccharide $\mathrm{F} 4$ antigen belonged exclusively to the IgM class, even as late as 10 months after infection. Human sera cross-reacted widely with F4 antigen from heterologous serovars. The antibodies involved in leptospiral agglutination were mainly $\operatorname{IgM}$, but some patients also produced IgG agglutinins. The titres of IgM agglutinins were higher than those of IgG agglutinins and persisted for many months, regardless of the presence or absence of IgG agglutinins. Both types of immunoglobulin from patients with serovar pomona infection protected hamsters against lethal infections with homologous leptospires. The hamster-protective capacity of human sera correlated well with agglutinin titres. Sera from patients infected with serovars other than pomona protected hamsters against challenge with pomona only if they contained agglutinins to that organism.

This work was supported by a grant from the National Health and Medical Research Council, Canberra. The experiments involving hamsters were conducted at the National Health Institute, New Zealand, by one of the authors (B. Adler) who is grateful to the Director-General of Health for permission to publish this paper. We acknowledge the technical assistance of J. Richardson and G. Parker.

\section{REFERENCES}

AdLeR, B. AND FAINE, S. 1976. Susceptibility of mice treated with cyclophosphamide to lethal infection with Leptospira interrogans serovar pomona. Infect. Immun., 14, 703.

AdLER, B. AND FAINE, S. 1978. Serological and protective antibody responses of rabbits to leptospiral antigens. J. Med. Microbiol., 11, 401.

Alston, J. M. AND BRoOM, J. C. 1958. Leptospirosis in man and animals, Edinburgh.

BABudier1, B. 1961. Laboratory diagnosis of leptospirosis. Bull. Wld Hlth Org., 24, 45.

Borg-Petersen, C. 1953. In Symposium on the leptospiroses. Walter Reed Army Medical Center, Med. Sci. Publ. No. 1, p. 174.

Chang, R. S. AND McComb, D. E. 1954. Erythrocyte sensitizing substances from five strains of Leptospirae. Am. J. trop. Med. Hyg., 3, 481.

Christmas, B. W., Bragger, J. M. AND Till, D. G. 1974. Dairyfarm fever in New Zealand: isolation of L. pomona and L. hardjo from a local outbreak. N.Z. Med. J., 79, 904.

Combiescu, D., Sturdza, N., Sefer, M. AND KlipPer, A. 1960. Évolution des agglutinines dans les leptospiroses humaines. Valeur de la réaction d'agglutination-lyse dans la détermination du type étiologique. Archs roum. Path. exp. Microbiol., 19, 201. 
Cox, C. D. 1955. Hemolysis of sheep erythrocytes sensitized with leptospiral extracts. Proc. Soc. exp. Biol. Med., 90, 610.

Cox, C. D., Alexander, A. D. AND MurPhy, L. C. 1957. Evaluation of the hemolytic test in the serodiagnosis of human leptospirosis. J. infect. Dis., 101, 210.

Crawford, R. P. 1972. Molecular characteristics of antibody detected by the microscopic agglutination test in serum of guinea pigs with leptospirosis. Am.J. vet. Res., 33, 1507.

FAINe, S., Adler, B. AND Palrt, A. 1974. Chemical serological and biological properties of a serotype-specific polysaccharide antigen in Leptospira. Aust. J. exp. Biol. med. Sci., 52, 311.

GraVes, S. AND FaINe, S. 1970. Antileptospiral agglutinins produced in rabbits. Bull. Wld Hlth Org., 43, 579.

hartmann, L., Filliti-wurmser, S., Jaquot-armand, Y., Mailloux, M., Hurez, D. AND FAUVERT, R. 1964. Nature macromoleculaire d'un anticorps de la leptospirose australis. Biochim. biophys. Acta, 82, 249.

Johnson, R. C., BEY, R. F. AND AURAN, N. E. 1975. The immunogenic properties of the outer envelope of parasitic leptospires. National Symposium on Leptospirosis, Leptospira and other Spirochaeta, Bucharest, abst.

Johnson, R. C. AND HARRIS, V. G. 1967. Differentiation of pathogenic and saprophytic leptospires. I. Growth at low temperatures. J. Bact, 94, 27.

McComb, D. E., Smith, D. J. W., Coffin, D. L., MacReady, R. A. and Chang, R. S. 1957. The use of erythrocyte sensitizing substance in the diagnosis of leptospiroses. I. The sensitized erythrocyte agglutination test. Am. J. trop. Med. Hyg. 6, 90.

Morris, J. A. AND HussainI, S. N. 1974. Characterization of the antibodies detected by the microscopic agglutination test for bovine leptospirosis. J. Hyg., Camb., 73, 425.

Morsi, H. M., ShIBley, G. P. AND STROTHER, H. L. 1973. Antibody response of swine to Leptospira canicola and Leptospira icterohaemorrhagiae. Am.J. vet. Res., 34, 1253.

Negi, S. K., MYers, W. L. AND SEgRe, D. 1971. Antibody response of cattle to Leptospira pomona: response as measured by hemagglutination, microscopic agglutination and hamster protection tests. Am. J. vet. Res., 32, 1915.

Palit, A. and Gulasekharam, J. 1973. Genus-specific leptospiral antigen and its possible use in laboratory diagnosis. J. clin. Path., 26, 7.

PIKe, R. M. 1967. Antibody heterogeneity and serological reactions. Bact. Rev., 31, 157.

Pike, R. M., McBrayer, H. L., Schulze, M. L. AND Chandler, C. H. 1965. Chromatographic analysis and sulfhydryl sensitivity of antileptospira agglutinins in rabbit and human sera. Proc. Soc. exp. Biol. Med., 120, 786.

SCHÜFFNER, W. AND MOCHTAR, A. 1926-27. Versuche zur Aufteilung von Leptospirenstämmen, mit ein leitenden Bemerkungen über den Verlauf von Agglutination und Lysis. Zentbl. Bakt. ParasitKde, I. Abt. Orig., 101, 405.

Sulzer, C. R., Glosser, J. W., Rogers, F., Jones, W. L. AND Frix, M. 1975. Evaluation of an indirect hemagglutination test for the diagnosis of human leptospirosis. J. clin. Microbiol., 2, 218.

Tong, M. J., Rosenberg, E. B., VotTeri, B. A. AND Che, C. T. 1971. Immunological response in leptospirosis. Report of three cases. Am. J. trop. Med. Hyg., $20,625$.

TURNer, L. H. 1968. Leptospirosis II. Trans. R. Soc. trop. Med. Hyg., 62, 880. 